\title{
Finite Element Model Fractional Steps Updating Strategy for Spatial Lattice Structures Based on Generalized Regression Neural Network
}

\author{
Caiwei Liu, Jijun Miao, and Changyong Zhao \\ College of Civil Engineering, Qingdao Technological University, Qingdao 266033, China \\ Correspondence should be addressed to Caiwei Liu; 03150053@163.com
}

Received 1 July 2015; Revised 22 September 2015; Accepted 28 September 2015

Academic Editor: Magd Abdel Wahab

Copyright (C) 2016 Caiwei Liu et al. This is an open access article distributed under the Creative Commons Attribution License, which permits unrestricted use, distribution, and reproduction in any medium, provided the original work is properly cited.

In order to get a more accurate finite element model of a spatial lattice structure with bolt-ball joints for health monitoring, a method of modifying the bolt-ball joint stiffness coefficient was proposed. Firstly, the beam element with adjustable stiffness was used in the joint zone in this paper to reveal the semirigid characteristic of the joint. Secondly, the value of stiffness reduction factor $\left(a_{r}\right)$ was limited in the range of $[0.2,0.8]$ and the reference value $\left(a_{r 0}\right)$ of it was suggested to be 0.5 based on referenced literatures. Finally, the finite element model fractional steps updating strategy based on neural network technique was applied and the limited measuring point information was used to form the network input parameter. A single-layer latticed cylindrical shell model with 157 joints and 414 tubes was used in a shaking TABLE test. Based on the measured modal data, the presented method was verified. The results show that this model updating technique can reflect the true dynamic characters of the shell structure better. Moreover, the neural network can be simplified considerably by using this algorithm. The method can be used for model updating of a latticed shell with bolt-ball joints and has great value in engineering practice.

\section{Introduction}

Structural health monitoring is becoming increasingly important, not only in the prevention of catastrophic failures, but also in improving maintainability of aerospace, civil, and mechanical infrastructure. An improved understanding of the capacity and performance of a structure within its serviceability limit states is a critical need and most often implemented by means of structural identification (STI) [1].

A typical STI application involves the determination of the modified parameters of a structure and subsequent revision of its finite element model accordingly, such that the improved (calibrated) model accurately simulates the response of the structure, as verified from field measurements [2]. It is crucial that the models are calibrated through STI procedures to permit reliable simulations and genuine performance assessments.

A good finite element model that accurately predicts the dynamic response of an actual structure is a prerequisite for many damage detection techniques. Structural model updating refines an analytical model using test data to better predict the dynamic behavior of a structure. In the last decade, finite element model updating has been intensively researched, and robust methods are now available to calculate adequate values of the selected model parameters from experimental data. Artificial neural networks (ANNs) have been successfully implemented in STI and model updating studies in the literature [3-6].

In real engineering projects, especially in long-span spatial structures, there are plenty of tubes and nodes; therefore, the number of parameters that need to be updated is large, which causes increase in the processing time and reduces the efficiency and accuracy of prediction, and the samples would lead to data explosion when training the ANN [7].

In order to solve this problem, fractional steps model updating method for different engineering structures is proposed. Both the substructure method and radial basis function neural network (RBF) were introduced into finite element model updating of single pylon cable-stayed bridges [4], which simplified the process of model updating and 


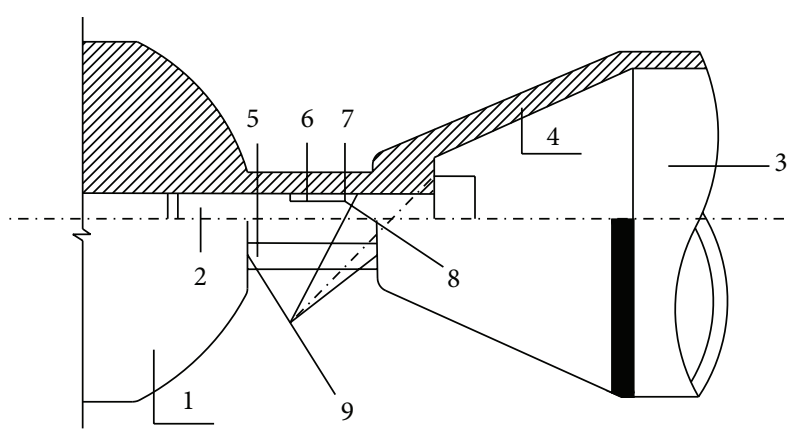

FIGURE 1: Bolt-ball joint with ball (1), ball (2), high strength bolt (3), steel tube (4), end cone (5), sleeve (6), slot (7), dowel pin (8), and deep-rich (9) contact surfaces.

greatly improved the updating efficiency. A stepwise model updating method based on substructures and ANN was presented by $\mathrm{He}$ et al. [6]. A single-layer reticulated dome was first divided into multilayer substructures, and model updating was then done step by step according to appropriate damage factors. However, only the elasticity modulus of tubes was updated.

There are also researches on updating the finite element models of structures with semirigid joints. In order to consider both the moment-rotation and shear-displacement characteristics of such joints, a hybrid finite element was proposed by Liu et al. [8]. Both of the characteristics of semirigid joints could be simulated by this element. However, due to the complicated operation, it is inconvenient for engineering application.

With the major advantages of material saving, aesthetic appearance, high construction speed, and high fabrication accuracy, single-layer latticed shells with bolt-ball joints are outstanding spatial structures [9]. Figure 1 illustrates the detailed structure.

Four types of fine bolt-ball joint finite element models were developed according to joints in a shaking TABLE experiment model of a single-layer latticed cylindrical shell with 157 joints and 414 tubes [10]. The friction coefficient and contact were used to simulate the sliding between the high-strength bolts and steel ball. The influences of the dowel pin and pretightening force were ignored. The $M-\theta$ (bending moment and rotating angle) curve of the bolt-ball connections was obtained. The connection was simplified to a joint element with the same inertia moment $I$; the elastic modulus of the joint element was determined from the obtained curve.

When the friction coefficient and contact parameters were determined according to the results of repeated trials, the stiffness reduction factors of the four types of bolt-ball joints were obtained (i.e., 0.524, 0.466, 0.317, and 0.122). By using these factors, sound model updating results could be determined. However, this method is still not practical for engineering applications, as the modeling process is complicated, even with a number of stiffness reduction factors and simplified assumptions. In fact, there is a difference in the bolt tightness due to manual operation, which cannot be simulated correctly by this model.
The spatial lattice structure with bolt-ball joints has the characteristics of modeling difficulty and plenty of tubes and nodes. In order to get a more accurate finite element model of a spatial lattice structure with bolt-ball joints for health monitoring, a new method that is suitable for the spatial lattice structure was proposed in this paper and then was used to analyze experimental results of a singlelayer latticed cylindrical shell model with 157 joints and 414 tubes tested using a shaking TABLE. Based on the measured modal data, the presented method was verified. The results show that this model updating technique can reflect the true dynamic characters of the shell structure better. Moreover, use of the general regression neural networks (GRNN) for model updating can be simplified considerably by using this approach. The method can be used for model updating of a latticed shell with bolt-ball joints and has great value in engineering practice.

\section{Construction of Semirigid Model}

In analysis of space structures, conventional designs and analyses of lattice shell structures are based on such an assumption that the connection behaves as either a pinned or rigid joint. However, the joints in most of the space structures are semirigid and their actual behaviors do not conform to either of the two extremities. A bolt-ball joint system with partial bend-bearing capacity is a typical semirigid joint system, which may be the main reason for the large difference in the results from dynamic testing and numerical simulation.

The accuracy of the finite element model of a spatial lattice structure depends on the connection mode between the tubes and the bolt ball. Figure 1 illustrates the detailed structure. The beam element with adjustable stiffness is used in the joint zone to reveal the semirigid characteristic of the joint, as shown in Figure 2. And then take the boundary conditions, the load cases, material property, and so on into consideration; the finite element model is finally constructed, which is called a semirigid model in this paper. $L$ is the length of the element; $a$ is the stiffness reduction factor (the joint element-member element stiffness ratio), where $0<a<1$. $E I$ is the element stiffness of the member; $a E I$ is the element stiffness of the joint, with coefficient $a$ defined considering the friction coefficient, contact parameters, and prestress force.

\section{Updating Strategy Basing on GRNN}

3.1. ANN and GRNN. An ANN is a biologically inspired information processing system that mimics a biological nerve system to exploit the imprecision and uncertainty of problems for deriving valuable and robust models [11]. It is composed of a large number of highly interconnected processing elements that are tied together with weighted connections and work in parallel to solve specific problems.

ANNs are particularly suitable for situations where conventional constitutive modeling may be insufficient, tedious, or time consuming. One of their distinctive characteristics is the ability to learn even in cases of erroneous, incomplete, or fuzzy data and make a reliable prediction on datasets not previously seen $[12,13]$. Their unique learning and prediction 


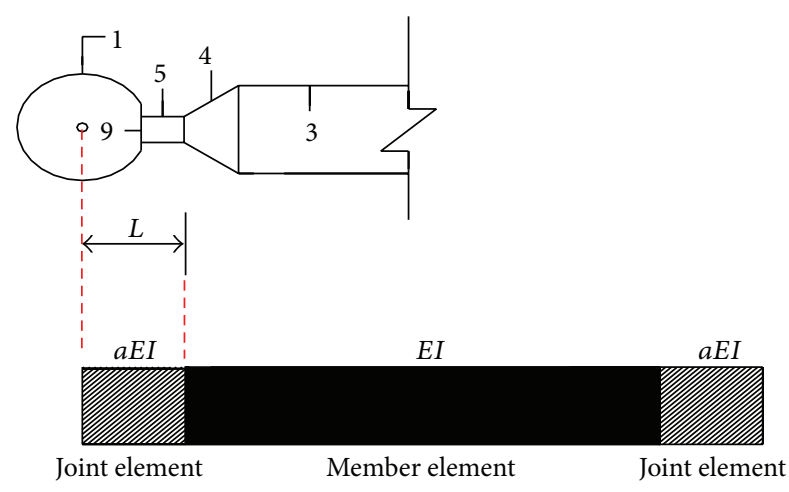

FIGURE 2: Joint element with adjustable stiffness parameters.

characteristics make them a popular tool for modeling problems encountered in various applications of civil and structural engineering.

GRNN has been proposed by Specht [14]. It uses a radial basis activation function, requires less training parameters, is difficult to become the local minimum, and is probabilistic compared to the traditional ANN $[15,16]$. GRNN is often a three-layer network in which there must be one hidden neuron for each training pattern. The GRNN is a type of supervised network and also trains quickly on sparse datasets, but rather than categorizing them, GRNN applications are able to create continuous valued outputs [17]. A principal advantage of GRNN is that they involve a one-pass learning algorithm and are consequently much faster to train than the well-known back-propagation paradigm [14]. Furthermore, they differ from classical neural networks in that every weight is replaced by a distribution of weights. GRNN is related to the RBF, based on a standard statistical technique called Gaussian kernel regression [18].

3.2. Determination of GRNN Input Parameters. A structure's natural frequency can be readily obtained by testing, and the value is relatively accurate. The frequency can reflect the dynamic characteristics of the whole structure; therefore, it is a good input parameter in model updating based on ANN [19]. However, the change of mode shape is very sensitive to structural damage, which is critical when a large spatial lattice structure is undergoing damage detection. Therefore, it is necessary to adopt a reasonable combination of frequency and mode as the input parameters according to the characteristics of a spatial lattice structure. The updated model requires that the natural frequency be close to the measured data and that the modal assurance criterion (MAC) value should be close to 1 as possible [1].

In this paper, the combined parameters of frequency and mode (CPFM) are adopted as the GRNN input as follows:

$$
\begin{aligned}
& \text { CPFM } \\
& \quad=\left\{\mathrm{FRE}_{1}, \mathrm{FRE}_{2}, \ldots, \mathrm{FRE}_{m} ; \mathrm{DF}_{1}, \mathrm{DF}_{2}, \ldots, \mathrm{DF}_{n}\right\},
\end{aligned}
$$

where $m$ is the order of the frequency; $n$ is the order of the mode shape; $\operatorname{FRE}_{i}(i=1,2, \ldots, m)$ is the $i$ th order frequency; and $\mathrm{DF}_{i}=\left(\varphi_{i 1}, \varphi_{i 1}, \ldots, \varphi_{i q},\right)$ and is the normalized modal

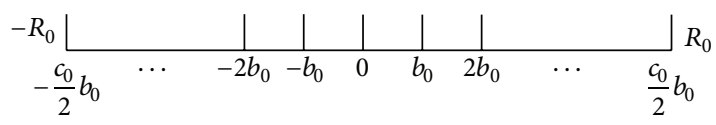

Figure 3: Variation ranges and steps.

vector of the $q$ measured degrees of freedom in the $i$ th order mode, which is calculated with the following formula:

$$
\varphi_{i j}=\frac{\varphi_{i j}}{\left(\varphi_{i j}\right)_{\max }} \quad(j=1,2, \ldots, q),
$$

where $\varphi_{i j}$ is the $j$ th degree of freedom component in the $i$ th order mode.

For spatial lattice structures, the modes are complicated and dense, and the high order modes are difficult to excite and identify. Based on engineering testing, the order of the natural frequency should be less than the first five $(m \leq 5)$ and the modal shape should be less than the first three $(n \leq 3)$ to ensure the validity of the measured data [20]. A relatively simple vibration form in these ranges is preferable.

3.3. Fractional Step Updating Algorithm. The modal calculation of a spatial lattice structure with a semirigid joint was carried out with joint bending stiffness in different testing samples: that is, $a_{r 0} \times\left(1-\left(c_{0} / 2\right) b_{0}\right) E I_{r}, \ldots, a_{r 0} \times\left(1-b_{0}\right) E I_{r}$, $a_{r 0} \times 1.0 E I_{r}, a_{r 0} \times\left(1+b_{0}\right) E I_{r}, \ldots, a_{r 0} \times\left(1+\left(c_{0} / 2\right) b_{0}\right) E I_{r}$ $(r=1,2, \ldots, p)$, where $E I$ is the member element bending stiffness, $a_{r}(r=1,2, \ldots, p$, with $p$ as the number of corrected parameters) is the reference value of a stiffness reduction factor assumed to be $a_{r 0}, b_{0}$ is the step of training samples within the variation range of $\left[-R_{0}, R_{0}\right]\left(R_{0}<1\right)$, and $c_{0}$ is the number of steps, as shown in Figure 3.

Applying the calculated CPFM as the input of the GRNN, the corresponding joint stiffness reduction factor can be obtained as the output, and according to these values, the sample database for the relationship between the CPFM and the joint stiffness reduction factor can be established. The number of training samples is $\left(C_{\left(c_{0}+1\right)}^{1}\right)^{p}$, " $C_{0}+1$ " stands for the total numbers of certain corrected parameters, and "1" stands for one of the total numbers of certain corrected parameters, when a one-step updating algorithm (model updating in a single step) is adopted; however, the one-step updating algorithm induces a data explosion and decreases the nonlinear mapping capacity when it is applied to a spatial lattice structure with multiple parameters, although it is theoretically feasible.

The fractional steps updating algorithm is proposed in this paper, in order to alleviate the data explosion and mitigate the loss of mapping capacity. The detailed procedure of $n$th updating steps is described in the following paragraphs.

In the first updating, $a_{r}$ is set to $a_{r 0}$, the step of training samples is set to $b_{1}$ in the variation range of $\left[-R_{0}, R_{0}\right]\left(R_{0}<1\right)$, and $c_{1}$ is the number of steps. $a_{r 0} \times\left(1-\left(c_{1} / 2\right) b_{1}\right) E I_{r}, \ldots, a_{r 0} \times$ $\left(1-b_{1}\right) E I_{r}, a_{r 0} \times 1.0 E I_{r}$, and $a_{r 0} \times\left(1+b_{1}\right) E I_{r}, \ldots, a_{r 0} \times(1+$ $\left.\left(c_{1} / 2\right) b_{1}\right) E I_{r}$ (where $r=1,2, \ldots, p$ ) are put into the model of a spatial lattice structure with a semirigid joint; the modal calculation is then carried out. 
Sample database $A_{1}$ on the relationship between the CPFM and the joint element stiffness reduction factor is established. The calculated CPFM is the input of the GRNN, and the corresponding stiffness reduction factor of the joint is the output, which is used to train the GRNN. The CPFM input of the GRNN is obtained from the results based on the measured modal data of the spatial latticed structure, and the output is the initial updating result of the stiffness reduction factor, namely, $a_{r 1}$ (where $r=1,2, \ldots, p$ ).

In the second updating, the corresponding stiffness reduction factor is $a_{r 1}$, the step of training samples is set to $b_{2}$ within the variation range of $\left[-R_{1}, R_{1}\right]\left(R_{1}<R_{0}\right)$, and $c_{2}$ is the number of steps. $a_{r 1} \times\left(1-\left(c_{2} / 2\right) b_{2}\right) E I_{r}, \ldots, a_{r 1} \times\left(1-b_{2}\right) E I_{r}$, $a_{r 1} \times 1.0 E I_{r}$, and $a_{r 1} \times\left(1+b_{2}\right) E I_{r}, \ldots, a_{r 1} \times\left(1+\left(c_{2} / 2\right) b_{2}\right) E I_{r}$ are put into the semirigid model of the spatial lattice structure. The modal calculation is then carried out; sample database $A_{2}$ is established.

Similarly, the next updating results of the stiffness reduction factor, namely, $a_{r 2}$, can be obtained. This continues with $n$th updating steps and building sample database $A_{n}$ and the $n$th updating results of the stiffness reduction factor can be obtained, namely, $a_{r n}$.

When $a_{r 0}$ is in the range of $[-60 \%, 60 \%], b_{0}=0.1, c_{0}=12$, and $p=3$, the number of GRNN samples under a one-step algorithm is the value of $\left(C_{13}^{1}\right)^{3}$, which is 2197 . However, when the updating is divided into three steps, namely, $R_{0}=0.6, b_{1}=$ 0.6 , and $c_{1}=2 ; R_{1}=0.3, b_{2}=0.3$, and $c_{2}=2$; and $R_{2}=0.1$, $b_{3}=0.6$, and $c_{3}=2$, the number of GRNN samples in each step is $\left(C_{3}^{1}\right)^{3}$, that is, 27 . Thus, the total number of samples is $3 \times 3 \times C_{3}^{1}$, that is, 81 .

The ratio of the number of samples between the two types of algorithms is $0.0123(27 / 2197)$; the ratio of the total number of samples is 0.0369 (81/2197). Obviously, the fractional steps updating algorithm greatly reduces the number of training samples. Moreover, the advantages of the fractional steps updating algorithm are more significant when the number of parameters is larger (with increasing $p$ ).

3.4. Convergence Criteria and Flowchart. Based on engineering testing and numerical simulation, the minimum frequency error between the measured results and the theoretical results is at least up to $1 \%$ [21], to assess the effectiveness of the updated model; the convergence criterion was chosen as

$$
W\left(\omega_{(n-1)}^{i}, \omega_{n}^{i}\right)=\left|\frac{\omega_{n}^{i}-\omega_{(n-1)}^{i}}{\omega_{n}^{i}}\right|,
$$

where $\omega_{(n-1)}^{i}$ is the $i$ th order frequency value derived from the $(n-1)$ th updating and $\omega_{n}^{i}$ is the $i$ th order frequency value derived from the $n$th updating.

With $W\left(\omega_{(n-1)}^{i}, \omega_{n}^{i}\right)$ under the condition of $W\left(\omega_{(n-1)}^{i}\right.$, $\omega_{n}^{i}$ ) $\leq 1 \%$ (where $i$ is the needed order of the frequency), the updating is stopped with the corresponding $a_{r n}$ (where $r=1,2, \ldots, p)$ as the final updating results of the stiffness reduction factor. Otherwise, the $(n+1)$ th updating continues.

Theoretically, model updating approaches both in multisteps and in single step must meet the same convergence criterion, which indicates that these two approaches can attain the same accuracy.

The finite element model updating flowchart of the spatial lattice structure in consideration of the semirigid characteristics of the bolt-ball joint is shown in Figure 4.

\section{Finite Element Model Updating of Single- Layer Lattice Cylindrical Shell}

4.1. Correlation Analysis of Dynamic Test. The experiment test model of the single-layer lattice cylindrical shell (the test shell) with a length of $21.0 \mathrm{~m}$, a width of $3.0 \mathrm{~m}$, and a vector height of $0.75 \mathrm{~m}$ is shown in Figure 5. The test shell was constructed with a bolt ball; the stiffness of the connection was enhanced by increasing the size of the bolt and sleeve. There were 4 kinds of tubes and corresponding bolts, as shown in Table 1.

The bolt ball was a BS180 type; the 2 nd column space was selected as a typical one, considering a 7-column space and its similarity in the number and type of the tubes, and is shown in Figure 6. The experiment was done in an 8-seismic array, as shown in Figure 7, at Beijing University of Technology.

The BEAM44 of ANSYS was applied to the test shell. BEAM44 is a uniaxial element with tension, compression, torsion, and bending capabilities. The element has six degrees of freedom at each node: translations in the nodal $x, y$, and $z$ directions and rotations about the nodal $x-, y$-, and $z$-axes. To guarantee the precision of modal calculation, each tube was divided into five elements.

According to the modal results obtained from numerical simulation, the measuring point layout priority comprehensive scheduling method was used for the arrangement of the sensors [7]. The sensors were just placed in the $Y$ and $Z$ directions while no sensor was placed in the third direction for the fact that, through modal theory analysis, the vibration in the third direction is weak, as shown in Figure 5. The sinusoidal excitation method was used in the dynamic test. The first four natural frequencies and the first three modal shapes were tested with two electromagnetic exciters with output of $50 \mathrm{~N}$, two DF1010 ultra-low-frequency function generators, and two KD2701 power amplifiers over the length of the test model. The method of test data processing used in this paper can be seen in [7].

Usually during engineering design of single-layer reticulated shell model, the length of a tube is usually considered to be the distance from one bolt-ball center to another; the joint connection is assumed to be rigid, which is defined as the rigid model in this paper. The frequency rate $\left(E R\left(\omega_{i}^{t}, \omega_{i}^{c}\right)\right)$ and modal assurance criterion $\left(\operatorname{MAC}\left(\varphi_{i}^{t}, \varphi_{i}^{c}\right)\right)$ were adopted to assess rationality of the rigid model with the following expressions:

$$
\begin{aligned}
\operatorname{ER}\left(\omega_{i}^{t}, \omega_{i}^{c}\right) & =\left|\frac{\omega_{i}^{t}-\omega_{i}^{c}}{\omega_{i}^{t}}\right|, \\
\operatorname{MAC}\left(\varphi_{i}^{t}, \varphi_{i}^{c}\right) & =\frac{\left|\varphi_{i}^{t^{T}} \varphi_{i}^{c}\right|^{2}}{\left|\varphi_{i}^{t^{T}} \varphi_{i}^{t}\right|\left|\varphi_{i}^{c T} \varphi_{i}^{c}\right|},
\end{aligned}
$$




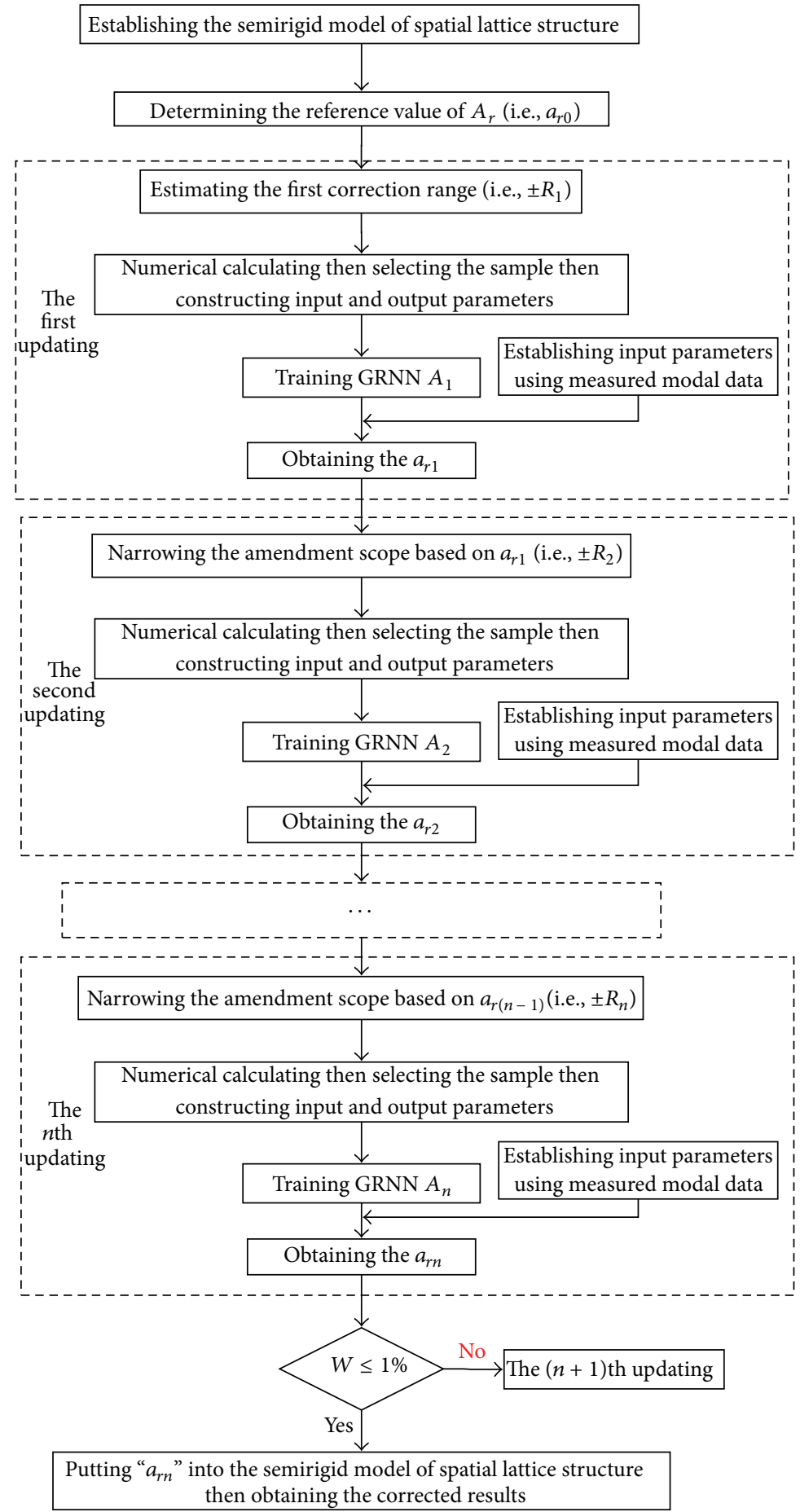

FIGURE 4: Flowchart of the finite element model fractional steps updating strategy.

TABLE 1: Tube types and specifications ( $\mathrm{mm})$.

\begin{tabular}{|c|c|c|c|c|}
\hline Tubes numbering & $1 \mathrm{~A} \quad 1 \mathrm{~B} \quad 1 \mathrm{C}$ & $2 \mathrm{~A}$ & $3 \mathrm{~A}$ & $4 \mathrm{~A}$ \\
\hline Tubes types & $\Phi 32 \times 2.15$ & $\Phi 48 \times 3.5$ & $\Phi 60 \times 3.5$ & $\Phi 89 \times 3.75$ \\
\hline Bolts & M16 & M24 & M24 & M27 \\
\hline
\end{tabular}

where $\omega_{i}^{t}(i=1,2, \ldots, n)$ is the actual measured natural frequency, $\varphi_{i}^{t}(i=1,2, \ldots, n)$ is the measured modal shapes, $\omega_{i}^{c}(i=1,2, \ldots, n)$ is the natural frequency obtained from the theoretical calculation, $\varphi_{i}^{c}(i=1,2, \ldots, n)$ is the modal shape obtained from the theoretical calculation, and $T$ is the matrix transposition. 


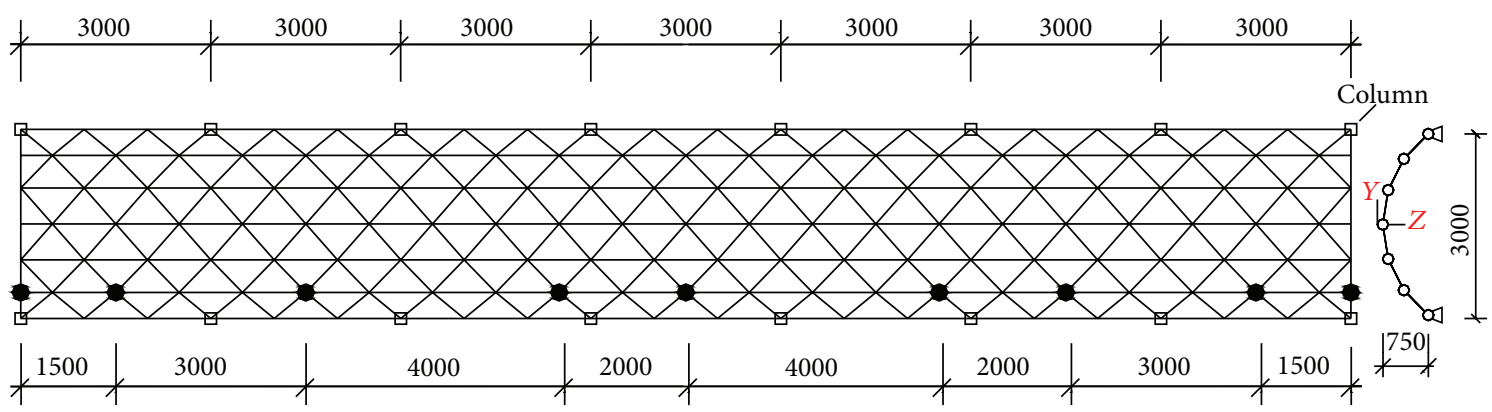

Figure 5: Test model of the reticulated shell $(\mathrm{mm})$, with the measuring point arrangement $(\bullet)$, and the node numbering from left to right is $1,25,46,60,88,109,130,151$, and 11 , respectively.

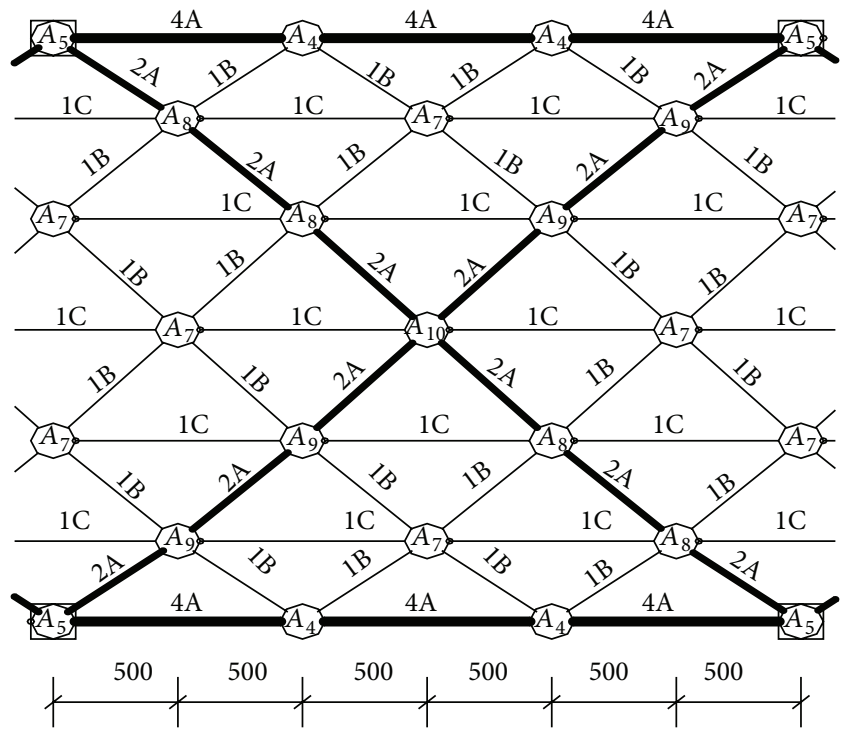

FIGURE 6: Structure's tubes.

TABLE 2: Comparison of the actual measured and rigid model results.

\begin{tabular}{lcccc}
\hline Modal & Measured values $(\mathrm{Hz})$ & Rigid model $(\mathrm{Hz})$ & ER $(\%)$ & MAC \\
\hline 1 & 6.62 & 7.86 & 18.81 & 0.77 \\
2 & 8.40 & 9.79 & 16.58 & 0.69 \\
3 & 10.1 & 13.18 & 30.50 & 0.51 \\
4 & 13.89 & 16.63 & 19.73 & - \\
\hline
\end{tabular}

The numerical model becomes more reliable as $\operatorname{ER}\left(\omega_{i}^{t}, \omega_{i}^{c}\right)$ approaches 0 and $\operatorname{MAC}\left(\varphi_{i}^{t}, \varphi_{i}^{c}\right)$ approaches 1 [20]. The minimum error of $16.58 \%$ (2nd order) can be seen in Table 2. The errors were induced by numerous factors, such as changes in support stiffness, and coordinate deviation of tubes and joints. However, ignoring the semirigid characteristic of bolt-ball joints is the main reason for the errors in the rigid model. Therefore, a fine numerical model was developed in this paper, by considering the semirigid characteristics of bolt-ball joints.
4.2. Corrected Parameters Determination. At present, many researches about spatial lattice structure have been reported. For example, by studying the welded spherical joints with beam element replacing nodes, Wang [22] concluded that good equivalent results were obtained when the ratio of the axial stiffness between tubes in the joint zone and the nonjoint zone was within the range of $45 \%$ to $50 \%$.

Much work has been done on the mechanical behavior of bolt-ball joints [23-26]. For example, the $M-\theta$ (bending moment and rotating angle) curve of bolt-ball joints was considered in an analysis of the seismic behavior of Kiewittype single-layer reticulated shells with bolt-ball joints [23]. The seismic internal force coefficients of K8 and K6 singlelayer spherical shells were proposed to be 1.80 and 1.44, respectively, which were bolt-ball joint stiffness reduction factors of 0.55 and 0.69 (i.e., reciprocals of 1.80 and 1.44), respectively.

Through an examination of the ultimate bearing capacities of bolt-ball joints (M20, M24, and M27) in K8 and K6 single-layer spherical shells, Fan et al. [26] concluded that the bolt-ball joint ultimate bearing capacity was approximately 0.4 to 0.8 times of that of a rigid joint.

In the literature $[10,22,23,26]$, the reference value of $a_{r}$ was $a_{r 0}$ (where $r=1,2,3,4$ ), which was set to 0.5 . In order to obtain a more realistic reduction factor for the test shell, the value of $a_{r}$ was limited in the range of [ $\left.-60 \%, 60 \%\right]$, that is, in the range of [0.2, 0.8]. Literature [27] shows that the corrected precision requirement could be satisfied when the value of $b_{i}$ $(i=1,2, \ldots, n)$ is 0.1 .

There are numerous parameters in the finite element model of a spatial lattice structure, and the sensitivity of each parameter to the dynamic characteristics is different. Therefore, if all of the parameters are taken into account in the model updating process, huge amounts of calculations and computer time are required, making a very inefficient model, and the results diverge. Accordingly, the relatively sensitive parameters are always chosen as the variables of the model updating problem; the dynamic sensitivity analysis of the parameters is turned into an effective way to solve the above problem [28].

There were four types of joint connections in the test shell model (i.e., M16-32 × 2.15, M24-48 × 3.5, M24-60 × 3.5 , and M27-89 $\times 3.75$ ). To obtain their dynamic sensitivity, 


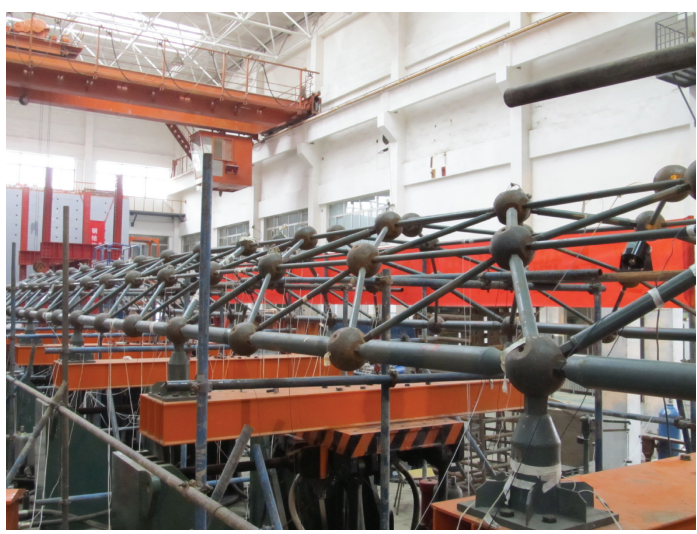

(a)

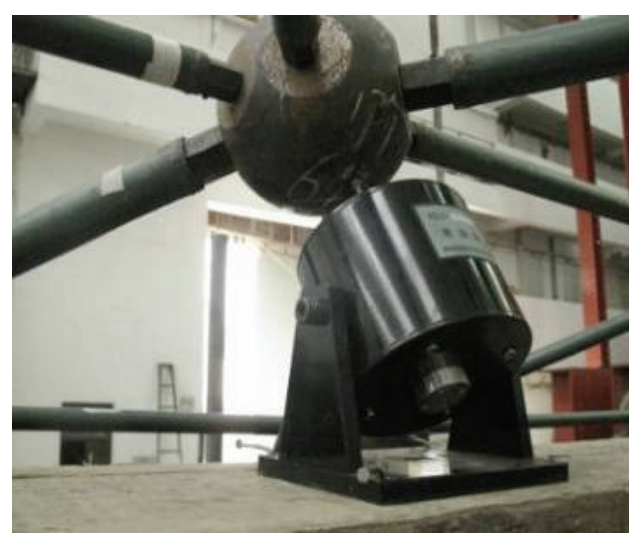

(b)

Figure 7: Test model field: (a) single-layer latticed cylindrical shell; (b) electromagnetic exciter using a $50 \mathrm{~N}$ output.

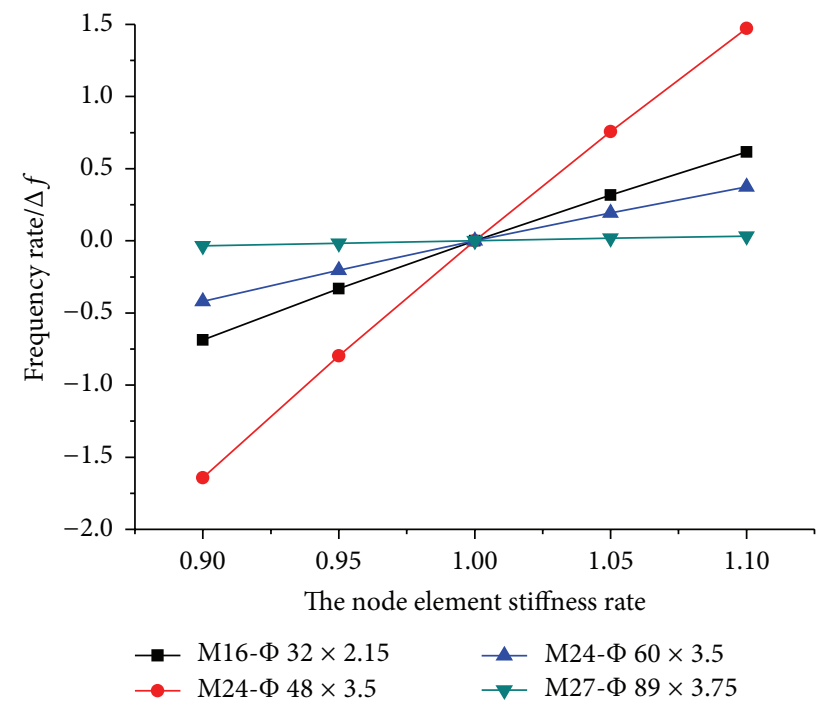

FIGURE 8: The influence of node element stiffness of connection in different types to the fundamental frequency.

frequency $f_{1.0}$ was derived from the joint element stiffness under $a_{r 0}$ (where $\left.r=1,2,3,4\right)=0.5$, frequency $f_{s}$ was derived from the joint element stiffness under $s \times a_{r 0}(s$ is magnification and equal to $0.9,0.95,1.05,1.1)$, and the formula of frequency rate was $\Delta f=\left(f_{s}-f_{1.0}\right) / f_{1.0}$. The calculated results are shown in Figure 8.

As shown in Figure 8, the joint element stiffness of M16$32 \times 2.15, \mathrm{M} 24-48 \times 3.5$, and M24-60 × 3.5 makes a considerable impact on the fundamental frequency of the test model; however, the joint element stiffness of M27- $\Phi 89 \times 3.75$ makes slight influence on it. The same law was evident for the frequency in the other orders. Therefore, the influence of M27- $\Phi 89 \times 3.75$ was omitted in the updating process; that is, its joint element stiffness reduction factor was considered as 0.5 .

In this paper, the bolt-ball joint element stiffness reduction factors of M16- $\Phi 32 \times 2.15$, M24- $448 \times 3.5$, and M24$\Phi 60 \times 3.5$ were corrected by GRNN. It was assumed that the same types of bolts had the same connection stiffness and that all connections were in sound condition; that is, the reference value of $a_{r 0}(r=1,2,3)$ was 0.5 and the optimized range was $[0.2,0.8]$.

4.3. Analysis of Corrected Results. According to Figure 4, the detailed procedures of fractional steps model updating were as follows:

(1) For the first updating, the values of $a_{r 0} \times(1-0.6) E I_{r}$, $a_{r 0} \times 1.0 E I_{r}$, and $a_{r 0} \times(1+0.6) E I_{r}(r=1,2,3)$ were put into the semirigid model of the test shell. The modal calculation was carried out with $a_{10}=a_{20}=$ $a_{30}=0.5, b_{1}=0.6$, and $c_{1}=2$ and the allowable variation range for $a_{r 0}$ was $[-60 \%, 60 \%]$. The CPFM (i.e., a $40 \times 1$ matrix) was derived from the first four natural frequencies and the first two modal shape components of the 18 sensors located on both $y$-and $z$-axes of the 9 positions (i.e., the solid black circles in Figure 5). Sample database $A_{1}$ for the relationship between the CPFM and the joint element stiffness reduction factor of the test shell was established.

Through the training of the GRNN with the CPFM, which was derived from the measured frequencies and modal shape components, with the input and the joint element stiffness reduction factors as the output, the joint element reduction factor of the test shell could be identified by the reverse neural network model. For further information and a complete formulation of GRNN as well as their detailed implementations steps, the reader is referred to a number of publications in the literature, such as $[1$, 15-18]. The initial correction results of the stiffness reduction factor were drawn $\left(a_{11}=0.721, a_{12}=\right.$ 0.573 , and $a_{13}=0.201$ ).

(2) Based on the results of the first updating, the results of the second updating were obtained $\left(a_{21}=0.658\right.$, $a_{22}=0.563$, and $\left.a_{23}=0.214\right)$ with $b_{2}=0.3, c_{2}=2$ and the allowable variation range of $[-30 \%, 30 \%]$ (i.e., $R_{1}=0.3$ ). 
TABLE 3: Results of updated frequency and $W\left(\omega_{(n-1)}^{i}, \omega_{n}^{i}\right) ; F_{n}^{i}$ is the $i$ th order frequency under the $n$th updating.

\begin{tabular}{lccccc}
\hline Modal & $F_{1}^{i}(\mathrm{~Hz})$ & $F_{2}^{i}(\mathrm{~Hz})$ & $F_{3}^{i}(\mathrm{~Hz})$ & $W\left(\omega_{1}^{i}, \omega_{2}^{i}\right)$ & $W\left(\omega_{2}^{i}, \omega_{3}^{i}\right)$ \\
\hline 1 & 6.78 & 6.70 & 6.65 & $1.25 \%$ & $0.75 \%$ \\
2 & 8.19 & 7.99 & 7.96 & $2.54 \%$ & $0.42 \%$ \\
3 & 11.48 & 10.89 & 10.82 & $5.35 \%$ & $0.66 \%$ \\
4 & 14.85 & 13.41 & 13.34 & $10.69 \%$ & $0.54 \%$ \\
\hline
\end{tabular}

(3) Based on the results of the second updating, the results of the third updating were obtained $\left(a_{31}=\right.$ $0.606, a_{32}=0.553$, and $\left.a_{33}=0.218\right)$ with $b_{3}=0.1$, $c_{3}=2$ and the allowable variation range of $[-10 \%$, $10 \%]$ (i.e., $R_{2}=0.1$ ).

The results of the updated frequency in the above steps and $W\left(\omega_{(n-1)}^{i}, \omega_{n}^{i}\right)$ are shown in Table 3. It is obvious that $W\left(\omega_{2}^{i}, \omega_{3}^{i}\right)<1 \%$ after the third updating, indicating that the results meet the convergence criterion after just 3 corrections. The results of the third updating and measured values are shown in Table 4, the comparison of frequency is shown in Figure 9, and the comparison of modal shapes is shown in Figure 10.

From Tables 3 and 4 and Figures 9 and 10, the following conclusions can be made:

(1) The trend of the correction results after each updating showed that they subsequently became closer to the measured value. The frequency errors of any order were reduced greatly, and the corrected results of the first-order mode were in good agreement with the measured results, accurately reflecting the true value of the dynamic characteristics of the test model. The correction method proposed in this paper was shown to be reasonable and effective.

(2) The characteristics of a huge structure and limited incentive energy in a spatial lattice structure led the lower order modes to be easily excited and the firstorder mode to be a simple translation movement; therefore, the measured results were in good agreement with the corrected ones. The third-order mode was complex and local vibration modal shapes may have existed, leading to the nonideality of the measured results; therefore, the large error in the thirdorder mode was because the inaccurate measured results were not taking into account in the GRNN input parameters.

(3) Although the error between the corrected and measured modes was greatly reduced, there were still some errors from other factors. For example, it was assumed that the geometry size and material properties of the tubes could be obtained accurately, and the stiffness correction of M27- $\$ 89 \times 3.75$, which had a low dynamic sensitivity, was not taken into account. GRNN may cause a little error when generalized with the experimental data. Moreover, the installation accuracy and testing environment during

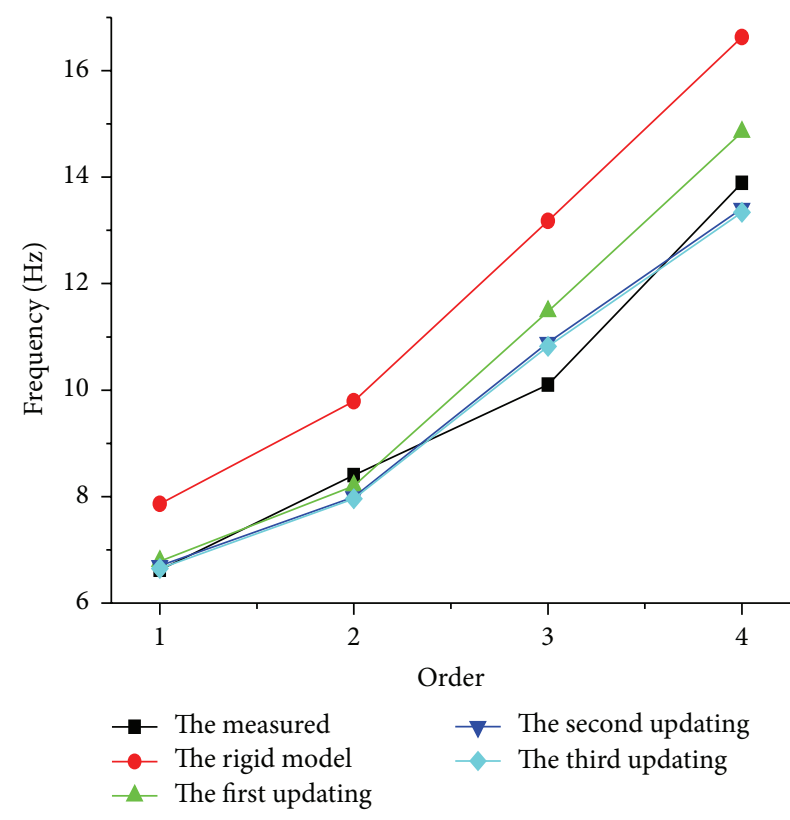

Figure 9: Comparison of frequency.

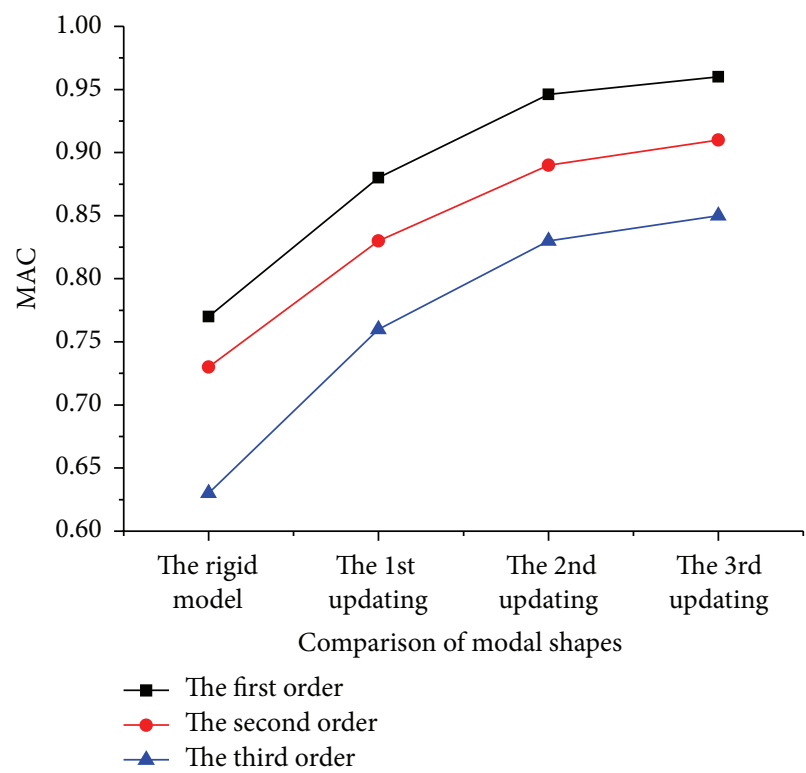

Figure 10: Comparison of modal shapes.

the test process could also have influenced the testing precision.

\section{Conclusions}

In this paper, the method that proposed the finite element model fractional steps updating strategy for a spatial lattice structure based on the GRNN authors has been proposed. It was applied to a test shell; the main conclusions are as follows:

(1) A new algorithm, the finite element model fractional steps updating strategy for a spatial lattice structure based on the GRNN, was proven to be reasonable 
TABLE 4: Results of model updating.

\begin{tabular}{|c|c|c|c|c|c|}
\hline \multirow{2}{*}{ Modal } & \multirow{2}{*}{$f$ (measured, Hz) } & \multirow{2}{*}{$f$ (updated, Hz) } & \multicolumn{2}{|c|}{ Correlation analysis } & \multirow{2}{*}{ Modal shapes } \\
\hline & & & $E R(\%)$ & MAC & \\
\hline 1 & 6.62 & 6.65 & 0.45 & 0.96 & $\begin{array}{l}\text { Type I, the whole structure displays translational motion } \\
\text { in the transverse direction. }\end{array}$ \\
\hline 2 & 8.40 & 7.96 & 5.23 & 0.91 & $\begin{array}{l}\text { Type II, the both ends of the structure display } \\
\text { nonsymmetric motion in the longitudinal direction. }\end{array}$ \\
\hline 3 & 10.10 & 10.82 & 7.13 & 0.85 & $\begin{array}{l}\text { Type III, the middle of the structure displays offset to one } \\
\text { side. } \\
\text { The both ends of the structure display symmetric motion } \\
\text { in the longitudinal direction. }\end{array}$ \\
\hline 4 & 13.89 & 13.34 & 3.96 & - & - \\
\hline
\end{tabular}

and effective when applied to the test shell. Compared with the correction methods of the traditional matrixes and parametric types, the new algorithm has the advantages of definite physical meaning, low computational complexity, strong robustness, and high accuracy.

(2) The new algorithm avoids the disadvantages of the GRNN, such as data explosion and decreased nonlinear mapping capacity. Therefore, it can be used easily and appropriately in model updating of a spatial lattice structure with the characteristics of multitudinous tubes and joints.

(3) To address the limited number of measured points on actual structures in project monitoring, the CPFM derived from low-order modal data of the limited measurement information of the structure was adopted as the input parameter of the GRNN and applied to the test shell. It was proven to be reasonable and effective. Therefore, the new algorithm is suitable for the correction of incomplete modal data.

(4) The method proposed in this paper is effective given the main effect factor (The semirigid characteristic of the bolt-ball joints) on the natural vibration characteristics. However, other effect factors, such as the elastic modulus of tubes and coordinate deviation of tubes and joints, also have some effect on it, so further studies considering more effect factors should be promoted.

\section{Conflict of Interests}

The authors declare that there is no conflict of interests regarding the publication of this paper.

\section{Acknowledgment}

This work is supported by the National Natural Science Foundation of China (Grant no. 51179081).

\section{References}

[1] O. Hasançebi and T. Dumlupinar, "Linear and nonlinear model updating of reinforced concrete T-beam bridges using artificial neural networks," Computers and Structures, vol. 119, pp. 1-11, 2013.

[2] D. Currie, N. Petrie, L. Mao, and Y. Lu, "A benchmark study of dynamic damage identification of plates," Proceedings of the Institution of Civil Engineers: Engineering and Computational Mechanics, vol. 165, no. 2, pp. 103-118, 2012.

[3] V. Meruane and J. Mahu, "Real-time structural damage assessment using artificial neural networks and antiresonant frequencies," Shock and Vibration, vol. 2014, Article ID 653279, 14 pages, 2014.

[4] D. H. Ding, Finite Element Model Updating of Single Pylon Cable-Stayed Bridges, Southwest Jiaotong University, Chengdu, China, 2012.

[5] L. Li, H. P. Zhu, and K. Z. Hong, "Experimental study on damage detection of frame structured based on artificial neural network algorithm," Journal of Vibration and Shock, vol. 25, pp. 107-109, 2006 (Chinese).

[6] H.-X. He, W.-M. Yan, and Z. Wang, "Stepwise model updating method based on substructures and GA-ANN," Engineering Mechanics, vol. 25, no. 4, pp. 99-105, 2008 (Chinese).

[7] C. W. Liu, Y. G. Zhang, and J. Z. Wu, "Experimental study on damage location of single-layer latticed cylindrical shell based on the dynamic testing," China Civil Engineering Journal, vol. 47, no. 6, pp. 9-17, 2014.

[8] Y. Liu, Z. D. Duan, and H. Liu, "Updating finite element model of structures with semi-rigid joints and boundary," in Smart Structures and Materials 2006: Sensors and Smart Structures Technologies for Civil, Mechanical, and Aerospace Systems, vol. 6174 of Proceedings of SPIE, pp. 61743L1-61743L13, 2006.

[9] H. H. Ma, F. Fan, Z. G. Cao, M. Y. Cui, and S. Z. Shen, "Numerical simulation of semi-rigid joints in single-layer dome structures," International Journal of Space Structures, vol. 52, pp. 3-18, 2011.

[10] Y. G. Zhang and Y. Z. Zhou, "Stiffness analysis and modeling simplification of the bolt-ball joints," in Proceedings of the 12th National Symposium on Modern Structural Engineering, Beijing, China, 2012.

[11] N. de Alcantara, "Identification of steel bars immersed in reinforced concrete based on experimental results of eddy current testing and artificial neural network analysis," Nondestructive Testing and Evaluation, vol. 28, no. 1, pp. 58-71, 2013.

[12] M. Y. Rafiq, G. Bugmann, and D. J. Easterbrook, "Neural network design for engineering applications," Computers \& Structures, vol. 79, no. 17, pp. 1541-1552, 2001. 
[13] A. A. Javadi and M. Rezania, "Applications of artificial intelligence and data mining techniques in soil modeling," Geomechanics and Engineering, vol. 1, no. 1, pp. 53-74, 2009.

[14] D. F. Specht, "A general regression neural network," IEEE Transactions on Neural Networks, vol. 2, no. 6, pp. 568-576, 1991.

[15] F. Doulati Ardejanii, R. Rooki, B. Jodieri Shokri, T. Eslam Kish, A. Aryafar, and P. Tourani, "Prediction of rare earth elements in neutral alkaline mine drainage from Razi coal mine, Golestan Province, northeast Iran, using general regression neural network," Journal of Environmental Engineering, vol. 139, no. 6, pp. 896-907, 2013.

[16] F. Han, D. W. Zhong, and J. Wang, "Model updating based on radial basis function neural network," Journal of Wuhan University of Science and Technology, vol. 34, pp. 115-118, 2011 (Chinese).

[17] S.-F. Jiang, C.-M. Zhang, and J. Yao, "Eigen-level data fusion model by integrating rough set and probabilistic neural network for structural damage detection," Advances in Structural Engineering, vol. 14, no. 2, pp. 333-350, 2011.

[18] S. Heddam, A. Bermad, and N. Dechemi, "Applications of radial-bas is function and generalized regression neural networks for modeling of coagulant dosage in a drinking watertreatment plant: comparative study," Journal of Environmental Engineering, vol. 137, pp. 1209-1214, 2011.

[19] H. F. Lam, J. M. Ko, and C. W. Wong, "Localization of damaged structural connections based on experimental modal and sensitivity analysis," Journal of Sound and Vibration, vol. 210, no. 1, pp. 91-114, 1998.

[20] Y. G. Zhang, C. W. Liu, and J. Z. Wu, “The application of the three-step location approach in damage identification of the spatial lattice structure," Disaster Advances, vol. 6, supplement 3, pp. 74-80, 2013.

[21] Y.-G. Zhang, C.-W. Liu, J.-Z. Wu, and T.-M. Peng, "Improved power spectrum peak method applied in the modal identification of spatial lattice structures," Journal of Vibration and Shock, vol. 32, no. 9, pp. 10-39, 2013 (Chinese).

[22] N. Wang, Study on fine model of single-layer cylindrical reticulated shell considering joint stiffness under strong earthquake [Ph.D. dissertation], Beijing University of Technology, 2013.

[23] F. Fan, M.-L. Wang, Z.-G. Cao, and S.-Z. Shen, "Seismic analysis of single-layer spherical reticulated shells with semi-rigid joint," Journal of Harbin Institute of Technology, vol. 41, no. 10, pp. 1419, 2009 (Chinese).

[24] A. López, I. Puente, and M. A. Serna, "Numerical model and experimental tests on single-layer latticed domes with semirigid joints," Computers and Structures, vol. 85, no. 7-8, pp. 360374, 2007.

[25] H. H. Ma, F. Fan, and S. Z. Shen, "Numerical parametric investigation of single-layer latticed domes with semirigid joints," International Journal of Space Structures, vol. 49, no. 158, pp. 99-110, 2008.

[26] F. Fan, H.-H. Ma, Z.-G. Cao, and S.-Z. Shen, "Direct estimation of critical load for single-layer reticulated domes with semirigid joints," International Journal of Space Structures, vol. 25, no. 1, pp. 15-24, 2010.

[27] Q. Xue, J. P. Hao, and Y. Zheng, "Research on steel frame parameters identification based on RBF neural networks," Journal of Xian University of Architecture and Technology (Natural Science Edition), vol. 43, pp. 192-197, 2011 (Chinese).

[28] D. Wang and Y.-Z. Zhu, "Mechanical characteristics analysis of sensitive parameters of long-span steel truss suspension bridge," Advanced Materials Research, vol. 250-253, pp. 3381-3386, 2011. 


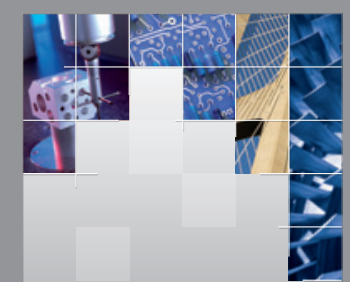

\section{Enfincering}
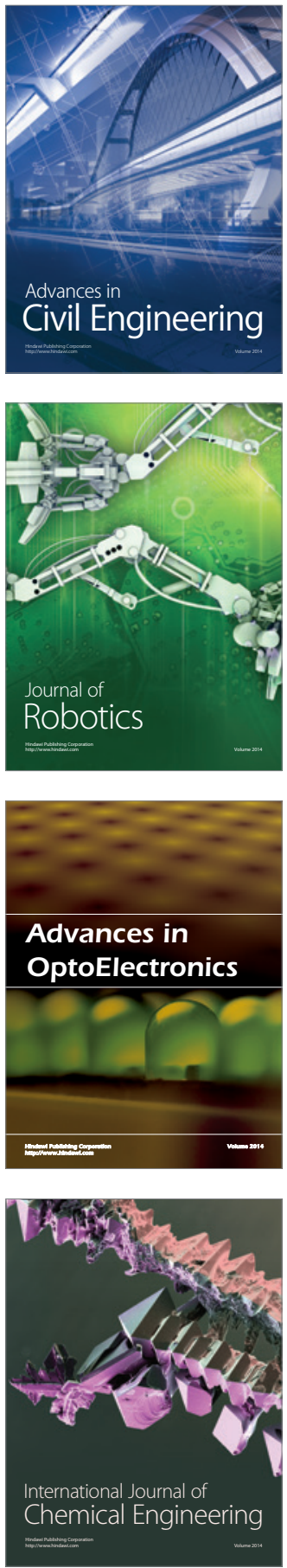

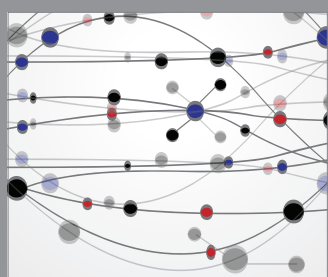

The Scientific World Journal

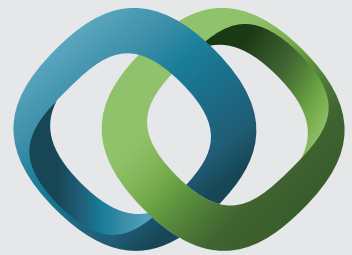

\section{Hindawi}

Submit your manuscripts at

http://www.hindawi.com
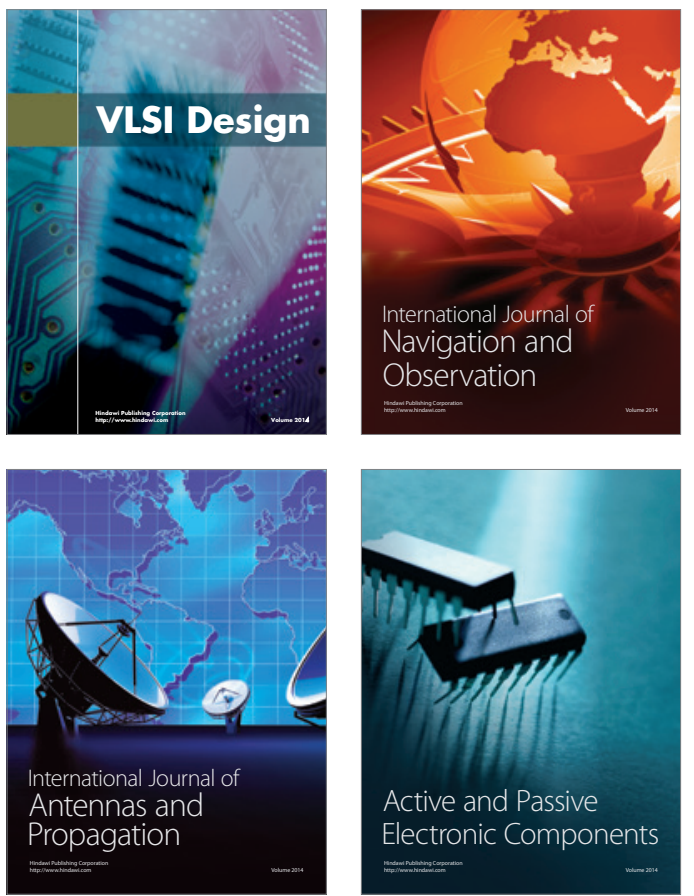
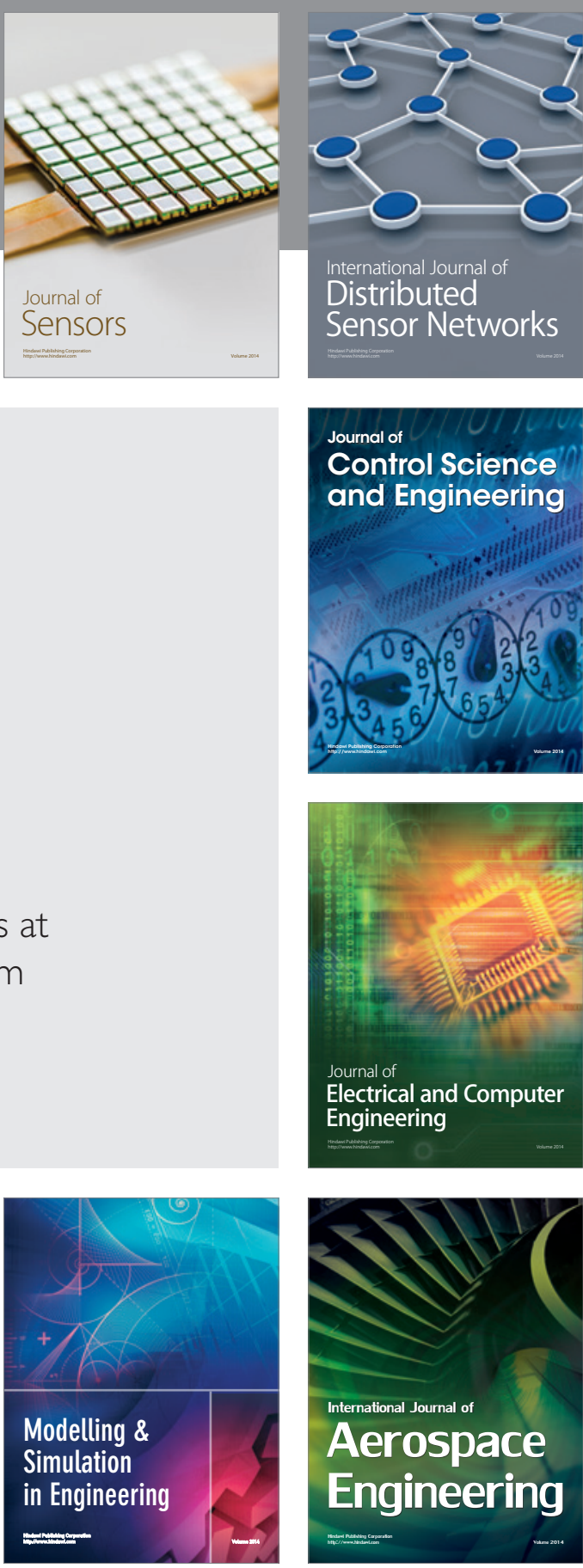

International Journal of

Distributed

Sensor Networks

Journal of

Control Science

and Engineering
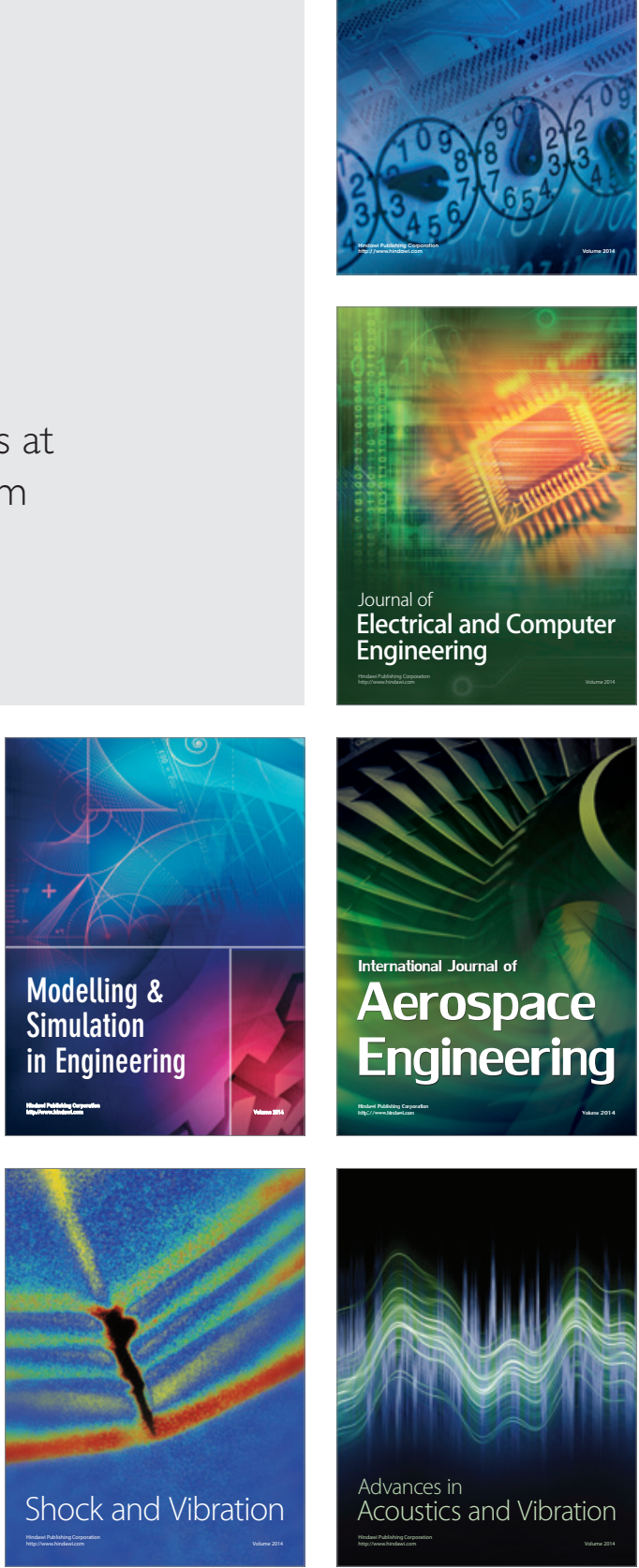